\title{
A systematic review of instruments assessing dimensions of distress among caregivers of adult and pediatric cancer patients
}

\author{
KIMBERSON TANCO, M.D., ${ }^{1 *}$ JI CHAN PARK, M.D., ${ }^{2 *}$ AGUSTINA CERANA, M.D., ${ }^{1}$ \\ AMY SISSON, M.S., M.L.S., ${ }^{3}$ NIKHIL SOBTI, M.S., ${ }^{4}$ AND EDUARDO BRUERA, M.D. ${ }^{1}$ \\ ${ }^{1}$ Department of Palliative Care and Rehabilitation Medicine, The University of Texas MD Anderson Cancer Center, \\ Houston, Texas \\ ${ }^{2}$ Division of Hematology/Oncology, Department of Internal Medicine, Daejeon St. Mary's Hospital, College of Medicine, \\ The Catholic University of Korea, Republic of Korea \\ ${ }^{3}$ Research Medical Library, The University of Texas MD Anderson Cancer Center, Houston, Texas \\ ${ }^{4}$ College of Arts and Sciences, Boston University, Boston, Massachusetts
}

(RECEIVEd October 22, 2015; ACCEPTED February 17, 2016)

\begin{abstract}
Objective: Caregivers of cancer patients face intense demands throughout the course of the disease, survivorship, and bereavement. Caregiver burden, needs, satisfaction, quality of life, and other significant areas of caregiving are not monitored regularly in the clinic setting, resulting in a need to address the availability and clinical effectiveness of cancer caregiver distress tools. This review aimed to determine the availability of cancer caregiver instruments, the variation of instruments between different domains of distress, and that between adult and pediatric cancer patient populations.

Method: A literature search was conducted using various databases from 1937 to 2013. Original articles on instruments were extracted separately if not included in the original literature search. The instruments were divided into different areas of caregiver distress and into adult versus pediatric populations. Psychometric data were also evaluated.

Results: A total of 5,541 articles were reviewed, and 135 articles $(2.4 \%)$ were accepted based on our inclusion criteria. Some 59 instruments were identified, which fell into the following categories: burden $(n=26,44 \%)$; satisfaction with healthcare delivery $(n=5,8.5 \%)$; needs $(n=$ $14,23.7 \%)$; quality of life $(n=9,15.3 \%)$; and other issues $(n=5,8.5 \%)$. The median number of items was 29 (4-125): 20/59 instruments (33.9\%) had $\leq 20$ items; $13(22 \%)$ had $\leq 20$ items and were psychometrically sound, with 12 of these $13(92.3 \%)$ being self-report questionnaires. There were 44 instruments $(74.6 \%)$ that measured caregiver distress for adult cancer patients and $15(25.4 \%)$ for caregivers of pediatric patients.

Significance of results: There is a significant number of cancer caregiver instruments that are self-reported, concise, and psychometrically sound, which makes them attractive for further research into their clinical use, outcomes, and effectiveness.
\end{abstract}

KEYWORDS: Cancer caregivers, Informal caregivers, Systematic review, Caregiver instruments, Caregiver tools

*Both authors contributed equally to this manuscript.

Address correspondence and reprint requests to: Kimberson Tanco, Department of Palliative Care and Rehabilitation Medicine, Unit 1414, The University of Texas MD Anderson Cancer Center, 1515 Holcombe Boulevard, Houston, Texas 77030. E-mail: kctanco@mdanderson.org.

\section{INTRODUCTION}

Cancer is a disease whose illness trajectory varies according to tumor site and grade (Aslett et al., 2009). The symptoms, treatments, and level of care needed by the cancer patient parallel this, and their disease course may range from a sudden and rapid decline to 
a prolonged course filled with sudden deteriorations in condition. Informal caregivers are expected to take on many roles: medical and financial decision maker, patient advocate, and care provider (National Cancer Institute, 2015). Moreover, in contrast to other diseases, cancer caregivers' tasks involve patients who enter the survivorship phase, including the psychological burden related to the possibility of disease recurrence. At the same time, the normal daily grind of their lives as well as that of the other family members they are already caring for continue, which exponentially increases their workload. The relationship between the patient and caregiver also plays a pivotal role, with a poor preexisting relationship as well as disagreements and conflicts within the family complicating care and decision making (Siminoff et al., 2006; Fried et al., 2003).

As the patient's illness becomes recurrent or terminal, caregivers are expected to provide increased care due to the decline in functional status and greater symptomatology. Caregivers have been found to report low quality of life and other health scores, even when compared to cancer patients (Weitzner et al., 1999a,b; Sales, 1992; Cameron et al., 2002; Glajchen, 2009; Kim \& Given, 2008). Physical, psychosocial, and financial complications occur, including insomnia (Hearson \& Clement, 2007), fatigue (Teel \& Press, 1999), cardiovascular disease (von Kanel et al., 2008), increased mortality (Christakis $\&$ Allison, 2006), increased rates of anxiety and depression (Given et al., 2004), and intensified feelings of fear and hopelessness (Dumont et al., 2006). In addition, due to the time-consuming aspect of caring for patients with advanced cancer, social isolation is likely to occur. Adequate social support, financial security, and work stability can offset this social impact (National Cancer Institute, 2015). Financial distress occurs due to accumulating copayments, insurance deductibles, travel costs, home care service expenses, and lost wages (National Cancer Institute, 2015; Glajchen, 2009; Yabroff et al., 2007). Early referral to palliative care has been shown to help improve the physical and psychological well-being of both patients and caregivers as well as enhance family satisfaction (Casarett et al., 2008; Gelfman et al., 2008). Spirituality has also been found to act as a safeguard against caregiver distress by helping caregivers gain a new perspective from the cancer experience and alleviate hopelessness, resulting in improved wellbeing (Ward et al., 1996; Kim et al., 2007).

Applebaum and Breitbart (2012) recently reviewed available interventions for informal caregivers, ranging from psychoeducation to complementary and alternative medicines. The majority of these interventions have shown benefits for informal caregivers. However, caregiver distress is still not monitored regularly, resulting in a need to address the availability of clinically applicable and effective caregiver tools that screen and detect any distress early on, which promotes more timely implementation of these interventions.

The objectives of our systematic review were: to determine the availability and adequacy of caregiver tools that were developed for cancer caregivers; to identify variations in the available cancer caregiver tools regarding different domains of distress, including such physical and psychosocial factors as caregiver needs, burden, and quality of life; and to identify the cancer caregiver tools that have been created for and utilized in the adult and pediatric populations.

\section{METHODS}

We conducted a literature search of all articles pertaining to tools for caregivers of cancer patients. During the initial search, restrictions were not placed in terms of language or publication type.

We employed the following databases to obtain relevant studies for this review (the original search was conducted up through October of 2013):

- MEDLINE (1946-present)

- EMBASE (1947-present)

- CINAHL (1937-present)

- Scopus (1996-present)

- PubMed ePubs (until October 2013)

We retrieved all articles through the strategy listed in Supplementary Appendix A for the MEDLINE search. The same strategy was adopted for the other databases. The methods and results section of each article were reviewed to determine if any reliability and validity tests had been conducted. In addition, we checked for references that might lead to further research on that tool to identify if reliability and validity were measured. See Tables 1-5 for details on the included studies.

An article was included if the instrument cited was designed specifically for caregivers of cancer patients to ensure that the instrument was originally designed with cancer caregiver distress in mind. If an instrument was created for a different population, including cancer patients but not caregivers, it was excluded. Since we intended to capture the whole caregiver experience, there were no restrictions set on the area that a tool assessed. Since there may be differences in the patient-caregiver relationship in the pediatric and adult cancer populations, we included instruments that measured caregivers of 
Table 1. Instruments focusing on caregiver burden

\begin{tabular}{|c|c|c|c|c|c|}
\hline Instrument name & Purpose/focus & Domains/sections & Original population & $\begin{array}{l}\text { No. of } \\
\text { items }\end{array}$ & Reliability/validity \\
\hline \multicolumn{6}{|l|}{ Adult Patients } \\
\hline $\begin{array}{l}\text { *Brief Assessment Scale } \\
\text { for Caregivers (BASC) } \\
\text { (Glajchen et al., 2005) }\end{array}$ & Caregiver burden & $\begin{array}{l}\text { Negative personal impact, positive } \\
\text { personal impact, other family } \\
\text { members, medical issues, concern } \\
\text { about loved one }\end{array}$ & $\begin{array}{l}\text { Caregivers of patients with chronic } \\
\text { illness (cancer 55\%, neurological } \\
15 \% \text {, psychiatric 12\%) }\end{array}$ & 14 & $\begin{array}{l}\text { Yes }(\alpha=0.70) / \text { yes } \\
\quad(\text { construct })\end{array}$ \\
\hline $\begin{array}{l}\text { *Caregiving } \\
\text { Consequences } \\
\text { Inventory (CCI) } \\
\text { (Sanjo et al., 2009) }\end{array}$ & $\begin{array}{l}\text { Family members' } \\
\text { perceptions of } \\
\text { caregiving } \\
\text { consequences }\end{array}$ & $\begin{array}{l}\text { Four reward domains (mastery, } \\
\text { appreciation for others, meaning in } \\
\text { life, reprioritization); one burden } \\
\text { domain }\end{array}$ & $\begin{array}{l}\text { Bereaved family members of cancer } \\
\text { patients }\end{array}$ & 16 & $\begin{array}{l}\text { Yes }(\alpha=0.78-0.93) / \\
\quad \text { yes (construct) }\end{array}$ \\
\hline $\begin{array}{l}\text { Appraisal of Caregiving } \\
\text { Scale (ACS) (Oberst et al., } \\
\text { 1989) }\end{array}$ & Caregiver burden & Harm/loss, threat, challenge, benign & $\begin{array}{l}\text { Family members of cancer patients } \\
\text { receiving radiotherapy }\end{array}$ & 53 & $\begin{array}{l}\text { Yes }(0.72-0.91) / \\
\text { yes }(\text { content })\end{array}$ \\
\hline $\begin{array}{l}\text { Cancer Caregiving Tasks, } \\
\text { Consequences and Needs } \\
\text { Questionnaire (CaTCoN) } \\
\text { (Lund et al., 2012) }\end{array}$ & $\begin{array}{l}\text { Caregiver tasks, } \\
\text { consequences and needs }\end{array}$ & $\begin{array}{l}\text { Caregiving tasks, caregiving consequences, } \\
\text { caregiver needs }\end{array}$ & Caregivers of cancer patients & 41 & Not found \\
\hline $\begin{array}{l}\text { Care Task Scale-Cancer } \\
\text { (CTS-C) [Taiwan] } \\
\text { (Chen et al., 2007) }\end{array}$ & Caregiver tasks & $\begin{array}{l}\text { Accompany patient and monitor care, } \\
\text { substitutive care for social and general } \\
\text { affairs, communication and emotional } \\
\text { care, mobility maintenance care }\end{array}$ & Spouse caregivers of cancer patients & 37 & $\begin{array}{l}\text { Yes }(\alpha=0.83-0.88) \\
\text { test-retest }(0.92- \\
0.94) / \text { yes } \\
\text { (construct) }\end{array}$ \\
\hline $\begin{array}{l}\text { Caregiver Assistance Scale } \\
\quad(\text { Cameron et al., 2002) }\end{array}$ & $\begin{array}{l}\text { Caregiver assistance on ADL, } \\
\text { iADL }\end{array}$ & None identified & $\begin{array}{l}\text { Caregivers of advanced cancer and } \\
\text { stroke patients }\end{array}$ & 17 & $\begin{array}{l}\text { Yes }(\alpha=0.87) / \text { not } \\
\quad \text { found }\end{array}$ \\
\hline $\begin{array}{l}\text { Caregiver Reaction } \\
\text { Assessment Scale (Given } \\
\text { et al., 1992) }\end{array}$ & $\begin{array}{l}\text { Reactions of various groups of } \\
\text { caregivers and changes in } \\
\text { reactions over time }\end{array}$ & $\begin{array}{l}\text { Caregiver esteem, lack of family support, } \\
\text { impact on finances, impact on schedule, } \\
\text { impact on health }\end{array}$ & $\begin{array}{l}\text { Exploratory portion: caregivers of elderly } \\
\text { patients with dementia/Alzheimer's } \\
\text { or various physical impairments/ } \\
\text { Confirmatory portion: caregivers of } \\
\text { cancer and Alzheimer's/dementia } \\
\text { patients }\end{array}$ & 24 & $\begin{array}{l}\text { Yes }(\alpha=0.91) / \text { yes } \\
\quad(\text { construct })\end{array}$ \\
\hline $\begin{array}{l}\text { Caregiver Self-Efficacy Scale } \\
\text { (CaSES) (Ugalde et al., } \\
\text { 2013) }\end{array}$ & Caregiver self- efficacy & $\begin{array}{l}\text { Resilience, self-maintenance, emotional } \\
\text { connectivity, instrumental caregiving }\end{array}$ & Caregivers of advanced cancer patients & 21 & $\begin{array}{l}\text { Yes }(\alpha=0.73-0.94) / \\
\text { yes (convergent, } \\
\text { divergent) }\end{array}$ \\
\hline $\begin{array}{l}\text { Caregiver's Communication } \\
\text { with the Patient about } \\
\text { Illness and Death (CCID) } \\
\text { (Bachner et al., 2008) }\end{array}$ & $\begin{array}{l}\text { Caregiver communication } \\
\text { with the patient about } \\
\text { illness and expected death } \\
\text { during the terminal stage }\end{array}$ & None identified & Caregivers of cancer patients & 8 & $\begin{array}{l}\text { Not found/yes } \\
\text { (convergent, } \\
\text { divergent) }\end{array}$ \\
\hline $\begin{array}{l}\text { Caregiving Impact Scale } \\
\text { (CIS) (Cameron et al., } \\
\text { 2002) }\end{array}$ & $\begin{array}{l}\text { Caregiver's experience of } \\
\text { lifestyle interference }\end{array}$ & $\begin{array}{l}\text { Health, diet, employment, active recreation, } \\
\text { passive recreation, finances, relationship } \\
\text { with partner, sex life, family relations, } \\
\text { other social relations, self-expression/ } \\
\text { improvement, religious expression, } \\
\text { community and civic involvement, } \\
\text { household responsibilities }\end{array}$ & $\begin{array}{l}\text { Caregivers of patients with incurable } \\
\text { cancer }\end{array}$ & 14 & $\begin{array}{l}\text { yes }(\alpha=0.87) / \text { Not } \\
\text { found }\end{array}$ \\
\hline $\begin{array}{l}\text { Demand-of-Illness Inventory } \\
\text { (DOII) (Haberman et al., } \\
\text { 1990) }\end{array}$ & Impact of disease & $\begin{array}{l}\text { Physical symptoms, personal meaning, } \\
\text { family functioning, social relationships, } \\
\text { self-image, monitoring symptoms, } \\
\text { treatment issues }\end{array}$ & $\begin{array}{l}\text { Family of mothers with breast cancer, } \\
\text { diabetes, and fibrocystic breast } \\
\text { changes }\end{array}$ & 125 & $\begin{array}{l}\text { Yes }(\alpha=0.96) / \text { yes } \\
\quad \text { (construct, } \\
\text { discriminant) }\end{array}$ \\
\hline
\end{tabular}


Family Appraisal of Caregiving Questionnaire for Palliative Care

(FACQ-PC) (Cooper et al. 2006)

Family Decision-Making Self-Efficacy Scale (Nolan et al., 2009)

Family's Difficulty Scale (FDS) (Ishii et al., 2012)

Parental Cancer Questionnaire (Levesque \& Maybery, 2014

Psychological Well-Being Scale for Caregiver (PWS-C) (Wu et al., 2010)

Prostate Care Questionnaire for Carers (PCQ-C) (Sinfield et al., 2009)

Suffering Scale of Family of Family suffering Patients with Terminal Cancer [Korean] (Kang, 2011)

Pediatric Patients

*Parental Worry and Attitudes Toward Attitudes Toward
Childhood Cancer (Duran, 2011)

Psychosocia Assessment Tool (Kazak et al., 2001)

\section{*Psychosocial} Assessment Tool 2.0 (Pai et al., 2008)
Family's appraisal of caregiving

\section{Fam}

amily member confidence in making decisions

Family's difficulties in caring for cancer patients at end of life at home

Adult children's experience of having a parent with cancer

Screen for psychological distress among family caregivers of advanced cancer patients in palliative care unit with prostate cancer

\section{Measure negative} attitudes of parents toward their child's cancer after treatment completion

Psychosocial risk in families for elevated distress in caring for child with cancer

Psychosocial risk in families for elevated distress in caring for a child with cance
Caregiver strain, positive caregiving appraisals, caregiver distress, family well-

F cance being

eing a surrogate, choosing treatments, accepting palliative care, meeting spiritua needs, maintaining family harmony, and communicating with health professionals

Burden of care, concerns about home care doctor, balance of work and care, patient's pain and condition, concerns about visiting nurse, concerns about home care service, relationship between family caregivers

and their families, funeral preparations

ALS (gradual decline) and pancreatic cancer (rapid decline) caregivers

Family's caring for cancer patient at end 29 of life at home

Adult children of parents with cancer strain

Social support, life meaning, emotional distress, caregiving inadequacy, hospital care

Carer experience when the patient is undergoing testing, when getting the diagnosis and making the treatment decision, during treatment, discharge and monitoring

Economical distress, loss of meaning in life, feeling of social isolation, emotional distress, burnout, guilty feeling, physical

Family caregivers of advanced cancer patients in a palliative care unit

Carers for men with prostate cancer discomfort

\section{Perseveration of parental worry (factor Parents of childhood cancer 1), psychosocial losses (factor 2) survivors}

Family resources, social support, child Families of newly diagnosed knowledge, child emotional and behavioral concerns, marital and

family problems, family beliefs

Family resources, social support, child knowledge, child emotional and behavioral concerns, marital and family problems, family beliefs pediatric oncology patients

Families of children with cancer

Yes $(\alpha=0.73-0.86)$ yes (construct)

Yes $(\alpha=0.91-0.95)$ test-retest $r=0.96) /$ yes (content)

Yes $(0.73-0.75) /$ yes convergent divergent)

Yes $(0.87-0.91) /$ yes No $(0.63-0.86$ [hospital care]) test-retest $(0.47$ $0.89) /$ yes (concurrent)

Yes $(\alpha=0.80-0.89)$ test-retest $(0.52$ $0.83) /$ yes (content)

Yes $(\alpha=0.93) /$ not found

Yes $(\alpha=0.91$, factor $1 ; \alpha=0.76$, factor 2)/yes (content)

Yes $(\alpha=0.83)$ testretest $(r=0.78$ 0.87)/yes (content)

Yes $(\alpha=0.81) /$ yes (content, riterion) 
Table 1. Continued

\begin{tabular}{|c|c|c|c|c|c|}
\hline Instrument name & Purpose/focus & Domains/sections & Original population & $\begin{array}{l}\text { No. of } \\
\text { items }\end{array}$ & Reliability/validity \\
\hline $\begin{array}{l}\text { Care of My Child With } \\
\text { Cancer (CMCC) (Wells } \\
\text { et al., 2002) }\end{array}$ & $\begin{array}{l}\text { Time and effort associated } \\
\text { with caring for a child with } \\
\text { cancer }\end{array}$ & None identified & $\begin{array}{l}\text { Primary caregivers of children with } \\
\text { cancer }\end{array}$ & 28 & $\begin{array}{l}\text { Yes }(0.93) \text { test-retest } \\
(0.90) / \text { yes (content, } \\
\text { construct) }\end{array}$ \\
\hline $\begin{array}{l}\text { Family Illness Beliefs } \\
\text { Inventory (FIBI) (Kazak } \\
\text { et al., 2004) }\end{array}$ & Cancer-related beliefs & $\begin{array}{l}\text { Treatment-related suffering, death and } \\
\text { devastation, caregiver competence, } \\
\text { connection, finding meaning }\end{array}$ & Parents of children with cancer & 41 & No/no \\
\hline $\begin{array}{l}\text { Life Situation Scale for } \\
\text { Parents (LSS-P) (Enskar } \\
\text { et al., 1997) }\end{array}$ & Parents' experiences & Care, well-being, social life, preparedness & Parents of children with cancer & 37 & $\begin{array}{l}\text { Yes }(\alpha=0.82) / \text { not } \\
\quad \text { found }\end{array}$ \\
\hline $\begin{array}{l}\text { Parental Coping Strategy } \\
\text { Inventory (PCSI) (Yeh, } \\
\text { 2001) }\end{array}$ & Parental coping & $\begin{array}{l}\text { Learning, struggling, interaction with } \\
\text { patient, interaction with spouse, } \\
\text { interaction with healthy sibling, emotion } \\
\text { support, information support, actual } \\
\text { support, maintaining stability, } \\
\text { maintaining an optimistic state of mind, } \\
\text { searching for spiritual meaning, } \\
\text { increasing religious activities }\end{array}$ & Parents of children with cancer & 48 & $\begin{array}{l}\text { Yes }(0.71-0.88) / \text { yes } \\
\quad \text { (construct) }\end{array}$ \\
\hline $\begin{array}{l}\text { Pediatric Inventory for } \\
\text { Parents (PIP) (Streisand } \\
\text { et al., 2001) }\end{array}$ & $\begin{array}{l}\text { Assess stress among parents } \\
\text { of children with a critical } \\
\text { illness }\end{array}$ & $\begin{array}{l}\text { Medical care, communication, role } \\
\text { functioning, emotional functioning }\end{array}$ & $\begin{array}{l}\text { Parents of children with a critical illness, } \\
\text { childhood cancer }\end{array}$ & 42 & $\begin{array}{l}\text { Yes (construct) } / \text { yes } \\
\quad(\alpha=0.95-0.96)\end{array}$ \\
\hline
\end{tabular}


Table 2. Instruments focusing on caregiver needs

\begin{tabular}{|c|c|c|c|c|c|}
\hline Instrument name & Purpose/focus & Domains/sections & Original population & $\begin{array}{l}\text { Number } \\
\text { of items }\end{array}$ & $\begin{array}{l}\text { Reliability/ } \\
\text { validity }\end{array}$ \\
\hline $\begin{array}{l}\text { Adult Patients } \\
\text { *Family } \\
\text { Inventory of } \\
\text { Needs } \\
\text { (Kristjanson } \\
\text { et al., 1995) }\end{array}$ & Caregiver needs & $\begin{array}{l}\text { Importance of } \\
\text { family care needs, } \\
\text { fulfillment of care } \\
\text { needs }\end{array}$ & $\begin{array}{l}\text { Families of } \\
\text { advanced cancer } \\
\text { patients }\end{array}$ & 20 & $\begin{array}{l}\text { Yes }(\alpha=0.83) / \\
\text { yes } \\
\text { (construct, } \\
\text { content) }\end{array}$ \\
\hline $\begin{array}{l}\text { ** Palliative } \\
\text { Care-Needs } \\
\text { Assessment } \\
\text { Tool (PC-NAT) } \\
\text { (Waller et al., } \\
\text { 2008) }\end{array}$ & $\begin{array}{l}\text { Patient and } \\
\text { caregiver } \\
\text { needs } \\
\text { determined by } \\
\text { health } \\
\text { professionals }\end{array}$ & $\begin{array}{l}\text { Patient well-being, } \\
\text { ability of } \\
\text { caregiver/family } \\
\text { to care for patient, } \\
\text { caregiver well- } \\
\text { being }\end{array}$ & $\begin{array}{l}\text { Health } \\
\text { professionals } \\
\text { treating } \\
\text { advanced cancer } \\
\text { patients and } \\
\text { their caregivers }\end{array}$ & 18 & $\begin{array}{l}\text { Yes } \\
\quad \text { (interrater)/ } \\
\text { yes (face, } \\
\text { content) }\end{array}$ \\
\hline $\begin{array}{l}\text { Caregivers Needs } \\
\text { Scale (Longman } \\
\text { et al., 1992) }\end{array}$ & Caregiver needs & $\begin{array}{l}\text { Personal care, activity } \\
\text { management, } \\
\text { involvement with } \\
\text { healthcare, work, } \\
\text { interpersonal } \\
\text { interaction, finance }\end{array}$ & $\begin{array}{l}\text { Caregivers of cancer } \\
\text { patients } \\
\text { undergoing } \\
\text { treatment }\end{array}$ & 90 & $\begin{array}{l}\text { Yes }(\alpha=0.80) / \\
\text { not found }\end{array}$ \\
\hline $\begin{array}{l}\text { Carer Support } \\
\text { Needs } \\
\text { Assessment Tool } \\
\text { (CSNAT) (Ewing } \\
\text { \& Grande 2013) }\end{array}$ & Caregiver needs & $\begin{array}{l}\text { Support to enable the } \\
\text { carer to provide } \\
\text { care, direct support } \\
\text { for carers }\end{array}$ & $\begin{array}{l}\text { Caregivers for } \\
\text { patients at end of } \\
\text { life, including } \\
\text { cancer }\end{array}$ & 50 & $\begin{array}{l}\text { Not found/yes } \\
\text { (face, content, } \\
\text { criterion) }\end{array}$ \\
\hline $\begin{array}{l}\text { Family Inventory } \\
\text { of Needs- } \\
\text { Husbands } \\
\text { (Kilpatrick et al., } \\
\text { 1998) }\end{array}$ & $\begin{array}{l}\text { Husband's } \\
\text { information } \\
\text { needs }\end{array}$ & $\begin{array}{l}\text { Surgical care needs, } \\
\text { communication with } \\
\text { doctor, family } \\
\text { relations, diagnosis/ } \\
\text { treatment specifics, } \\
\text { husband's } \\
\text { involvement }\end{array}$ & $\begin{array}{l}\text { Husbands of women } \\
\text { with breast cancer }\end{array}$ & 30 & $\begin{array}{c}\text { Yes }(\alpha=0.91- \\
0.93) / \text { yes } \\
\text { (content) }\end{array}$ \\
\hline $\begin{array}{l}\text { Family Needs } \\
\text { Assessment } \\
\text { (FNA) (Tringali, } \\
\text { 1986) }\end{array}$ & Caregiver needs & $\begin{array}{l}\text { Cognitive, emotional, } \\
\text { physical }\end{array}$ & $\begin{array}{l}\text { Family members of } \\
\text { cancer patients in } \\
\text { three different } \\
\text { phases of illness } \\
\text { (initial treatment, } \\
\text { recurrent disease, } \\
\text { follow-up } \\
\text { treatment) }\end{array}$ & 53 & $\begin{array}{l}\text { Not found/yes } \\
\quad \text { (content) }\end{array}$ \\
\hline $\begin{array}{l}\text { Home Caregiver } \\
\text { Need Survey } \\
\text { (Hileman et al., } \\
\text { 1992) }\end{array}$ & Caregiver needs & $\begin{array}{l}\text { Psychological, } \\
\text { informational, } \\
\text { patient care needs, } \\
\text { personal, spiritual, } \\
\text { household }\end{array}$ & $\begin{array}{l}\text { Home caregivers of } \\
\text { cancer patients }\end{array}$ & 90 & $\begin{array}{l}\text { Yes }(\alpha=0.85- \\
0.97) / \text { yes } \\
\text { (construct, } \\
\text { content) }\end{array}$ \\
\hline $\begin{array}{l}\text { Needs Assessment } \\
\text { of Family } \\
\text { Caregivers- } \\
\text { Cancer (NAFC- } \\
\text { C) (Kim et al., } \\
\text { 2010) }\end{array}$ & $\begin{array}{l}\text { Caregiver needs } \\
\text { during } \\
\text { survivorship } \\
\text { phase }\end{array}$ & $\begin{array}{l}\text { Cognitive/ } \\
\text { informational, } \\
\text { communication, } \\
\text { daily activity, } \\
\text { emotional, } \\
\text { financial/legal, } \\
\text { medical, social/ } \\
\text { relationship, } \\
\text { spiritual needs }\end{array}$ & Cancer caregivers & 27 & No/no \\
\hline $\begin{array}{l}\text { Needs Assessment } \\
\text { Tool-Caregivers } \\
\text { (NAT-C) } \\
\text { (Mitchell et al., } \\
\text { 2010) }\end{array}$ & Caregiver needs & $\begin{array}{l}\text { Information, practical } \\
\text { support, personal } \\
\text { health and well- } \\
\text { being, relationships, } \\
\text { meaning }\end{array}$ & Cancer caregivers & 33 & $\mathrm{No} /$ no \\
\hline
\end{tabular}


Table 2. Continued

\begin{tabular}{|c|c|c|c|c|c|}
\hline Instrument name & Purpose/focus & Domains/sections & Original population & $\begin{array}{l}\text { Number } \\
\text { of items }\end{array}$ & $\begin{array}{l}\text { Reliability/ } \\
\text { validity }\end{array}$ \\
\hline $\begin{array}{l}\text { Offspring Cancer } \\
\text { Needs } \\
\text { Instrument } \\
\text { (OCNI) } \\
\text { (Patterson et al., } \\
\text { 2011) }\end{array}$ & Unmet needs & $\begin{array}{l}\text { Information, family } \\
\text { issues, practical } \\
\text { assistance, time out, } \\
\text { feelings, support } \\
\text { (friends), support } \\
\text { (other young people) }\end{array}$ & $\begin{array}{l}\text { Young people }(12-24 \\
\text { years) impacted by } \\
\text { parental cancer }\end{array}$ & 47 & $\begin{array}{l}\text { Yes }(\alpha=0.89) \\
\text { test-retest } \\
\quad(r=0.73) / \text { yes } \\
\text { (construct) }\end{array}$ \\
\hline $\begin{array}{l}\text { Supportive Care } \\
\text { Needs Survey- } \\
\text { Partners and } \\
\text { Caregivers } \\
\text { (SCNS-P\&C) } \\
\text { (Girgis et al., } \\
\text { 2011) }\end{array}$ & Caregiver needs & $\begin{array}{l}\text { Healthcare service } \\
\text { needs, psychological } \\
\text { and emotional } \\
\text { needs, work and } \\
\text { social needs, } \\
\text { information needs }\end{array}$ & Cancer caregivers & 44 & $\begin{array}{c}\text { Yes }(\alpha=0.88- \\
0.94) / \text { partial } \\
\text { (construct) }\end{array}$ \\
\hline $\begin{array}{l}\text { Support Person } \\
\text { Unmet Needs } \\
\text { Survey (SPUNS) } \\
\text { (Campbell et al., } \\
\text { 2009) }\end{array}$ & Unmet needs & $\begin{array}{l}\text { Information and } \\
\text { relationships, } \\
\text { emotional needs, } \\
\text { personal needs, } \\
\text { work and finance, } \\
\text { healthcare access } \\
\text { and continuity, } \\
\text { worries about future }\end{array}$ & $\begin{array}{l}\text { Principal caregivers } \\
\text { of cancer survivors }\end{array}$ & 78 & $\begin{array}{l}\text { Yes }(\alpha>0.90) / \\
\text { yes (face, } \\
\text { content, } \\
\text { construct) }\end{array}$ \\
\hline \multicolumn{6}{|l|}{$\begin{array}{l}\text { Pediatric } \\
\text { Patients }\end{array}$} \\
\hline $\begin{array}{l}\text { *Family } \\
\text { Inventory of } \\
\text { Needs- } \\
\text { Pediatrics II } \\
\text { (FIN-PED II) } \\
\text { (Monterosso } \\
\text { et al., 2006) }\end{array}$ & Caregiver needs & $\begin{array}{l}\text { Importance of care } \\
\text { needs, extent to } \\
\text { which needs were } \\
\text { met, need for } \\
\text { further } \\
\text { information }\end{array}$ & $\begin{array}{l}\text { Parents of children } \\
\text { with cancer }\end{array}$ & 17 & $\begin{array}{l}\text { Yes }(\alpha=0.83- \\
98) \text { test- } \\
\text { retest } \\
(r=0.74- \\
\text { 0.94) } / \text { yes } \\
(\text { content })\end{array}$ \\
\hline $\begin{array}{l}\text { Family Inventory } \\
\text { of Needs- } \\
\text { Pediatrics (FIN- } \\
\text { PED) (Whiteley } \\
\text { et al., 1999) }\end{array}$ & Caregiver needs & $\begin{array}{l}\text { Importance of care } \\
\text { needs and extent to } \\
\text { which needs were } \\
\text { met }\end{array}$ & $\begin{array}{l}\text { Mothers of children } \\
\text { with cancer }\end{array}$ & 52 & $\begin{array}{l}\text { Yes }(\alpha=0.94) / \\
\quad \text { yes (construct) }\end{array}$ \\
\hline
\end{tabular}

*Self-report instruments with $\leq 20$ items, and positive reliability and validity.

**Healthcare provider a dministered instrument with $\leq 20$ items, and positive reliability and validity.

both adult and pediatric cancer patients and divided the instruments by population within the various areas of the caregiving experience.

The authors carried out a study selection to determine that all the articles met our inclusion criteria. Any disagreements about a particular study were resolved by discussion and consensus. Articles that were not studies on caregivers of cancer patients were excluded. Articles about cancer caregivers but with no standardized tools reported were also excluded. Articles that were only published as conference abstracts, research protocols, or theses, with no corresponding published manuscript, were also excluded. Finally, we also excluded articles for which no English translation was available.

The data were then extracted into the following areas of focus: purpose of the tool; dimensions measured; population originally developed for; number of items; and psychometric data. In order to allow these tools to be implemented in a clinical setting and avoid excessive burden to caregivers and clinicians in completing them, our team chose a cutoff of 20 items. Table 6 summarizes the distribution of tools and their number of items.

\section{Definition of Burden}

The term "burden" has been employed to define the impact on clinical and psychosocial factors within families. In Given's study on the Caregiver Reaction Assessment Scale, "caregiver burden" was defined as the physical, psychological, social, emotional, and financial problems experienced by family members (including both objective and subjective factors) (Given et al., 1992; Gwyther \& George, 1986; Montgomery et al., 1985). 
Table 3. Instruments focusing on satisfaction with care

\begin{tabular}{|c|c|c|c|c|c|}
\hline Instrument name & Purpose/focus & Domains/sections & Original population & Number of items & Reliability/validity \\
\hline \multicolumn{6}{|l|}{ Adult Patients } \\
\hline $\begin{array}{l}\text { *aregiver Evaluation of } \\
\text { Quality of End-of-Life } \\
\text { Care (CEQUEL) } \\
\text { (Higgins \& Prigerson, } \\
\text { 2013) }\end{array}$ & $\begin{array}{l}\text { Caregiver } \\
\text { satisfaction } \\
\text { with end-of-life } \\
\text { care }\end{array}$ & $\begin{array}{l}\text { Prolongation of death, perceived } \\
\text { suffering, shared decision } \\
\text { making, preparation for death }\end{array}$ & $\begin{array}{l}\text { Caregivers of } \\
\text { advanced cancer } \\
\text { patients }\end{array}$ & 13 & $\begin{array}{l}\text { Partial }(\alpha=0.52- \\
\text { 0.78) } / \text { yes } \\
(\text { convergent })\end{array}$ \\
\hline $\begin{array}{l}\text { *FAMCARE (Kristjanson, } \\
\text { 1993) }\end{array}$ & $\begin{array}{l}\text { Family } \\
\text { satisfaction } \\
\text { with advanced } \\
\text { cancer care }\end{array}$ & $\begin{array}{l}\text { Information giving, availability of } \\
\text { care, physical care, } \\
\text { psychosocial care }\end{array}$ & $\begin{array}{l}\text { Family members of } \\
\text { patients with } \\
\text { advanced cancer }\end{array}$ & 20 & $\begin{array}{l}\text { Yes }(\alpha=0.93) / \text { yes } \\
\quad(\text { criterion })\end{array}$ \\
\hline $\begin{array}{l}\text { Canadian Health Care } \\
\text { Evaluation Project } \\
\text { (CANHELP) } \\
\text { questionnaire (Heyland } \\
\text { et al., 2010) }\end{array}$ & $\begin{array}{l}\text { Patient and family } \\
\text { satisfaction with } \\
\text { end-of-life care }\end{array}$ & None identified & $\begin{array}{l}\text { Patient with advanced, } \\
\text { life-limiting illness } \\
\text { and their caregivers } \\
\text { (including metastatic } \\
\text { cancer) }\end{array}$ & $\begin{array}{l}38 \text { items (patient } \\
\text { version), } 40 \\
\text { items (family } \\
\text { caregiver } \\
\text { version) }\end{array}$ & $\begin{array}{l}\text { Yes }(\alpha=0.69-0.94) / \\
\quad \text { yes (construct) }\end{array}$ \\
\hline $\begin{array}{l}\text { Good Death Inventory } \\
\quad \text { (Miyashita et al., 2008) }\end{array}$ & Evaluate death & $\begin{array}{l}10 \text { core domains (Environmental } \\
\text { comfort, life completion, dying in a } \\
\text { favorite place, maintaining hope } \\
\text { and pleasure, independence, } \\
\text { physical and psychological comfort, } \\
\text { good relationship with medical staff, } \\
\text { not being a burden to others, good } \\
\text { relationship with family, being } \\
\text { respected as an individual); } 8 \\
\text { optional domains (religious and } \\
\text { spiritual comfort, receiving enough } \\
\text { treatment, control over the future, } \\
\text { feeling that one's life is worth living, } \\
\text { unawareness of death, pride and } \\
\text { beauty, natural death, preparation } \\
\text { for death) }\end{array}$ & $\begin{array}{l}\text { Bereaved family } \\
\text { members of cancer } \\
\text { patients }\end{array}$ & $\begin{array}{l}30 \text { (core domains) } \\
\quad+24 \text { (optional } \\
\quad \text { domains) }\end{array}$ & $\begin{array}{l}\text { Yes }(\alpha=0.74-0.95) \\
\text { test-retest } \\
(\mathrm{ICC}=0.38-0.72) / \\
\text { yes (concurrent) }\end{array}$ \\
\hline $\begin{array}{l}\text { Post Mortem } \\
\text { Questionnaire-Short } \\
\text { Form (QPM-SF) } \\
\text { (Partinico et al., 2014) }\end{array}$ & $\begin{array}{l}\text { Assess home and } \\
\text { inpatient hospice } \\
\text { care }\end{array}$ & $\begin{array}{l}\text { Integrated home care, hospice, } \\
\text { physical care, information, global } \\
\text { evaluation, needs }\end{array}$ & $\begin{array}{l}\text { Family caregivers of } \\
\text { cancer patients } \\
\text { receiving palliative } \\
\text { care }\end{array}$ & 37 & $\begin{array}{l}\text { Yes }(\alpha=0.85-0.99) \\
\text { test-retest } \\
\quad(\mathrm{ICC}=0.66-0.98) / \\
\text { none found }\end{array}$ \\
\hline $\begin{array}{l}\text { Pediatric Patients } \\
\text { None found }\end{array}$ & & & & & \\
\hline
\end{tabular}

*Self-report instruments with $\leq 20$ items and positive reliability and validity. 
Table 4. Instruments focusing on caregiver quality of life (QoL)

\begin{tabular}{|c|c|c|c|c|c|}
\hline Instrument name & Purpose/focus & Domains/sections & $\begin{array}{l}\text { Original } \\
\text { population }\end{array}$ & $\begin{array}{l}\text { Number } \\
\text { of items }\end{array}$ & $\begin{array}{l}\text { Reliability/ } \\
\text { validity }\end{array}$ \\
\hline $\begin{array}{l}\text { Adult Patients } \\
\text { *Caregiver Quality } \\
\text { of Life Index } \\
\text { (McMillan \& } \\
\text { Mahon, 1994) }\end{array}$ & QoL & $\begin{array}{l}\text { Emotional, social, } \\
\text { financial, physical }\end{array}$ & $\begin{array}{l}\text { Primary } \\
\text { caregivers of } \\
\text { cancer } \\
\text { patients in } \\
\text { hospice }\end{array}$ & 4 & $\begin{array}{l}\text { Yes }(\alpha=0.76- \\
0.88) / \text { yes } \\
(\text { content, } \\
\text { construct })\end{array}$ \\
\hline $\begin{array}{l}\text { *Family Pain } \\
\text { Questionnaire } \\
\text { (FPQ) (Ferrell } \\
\text { et al., 1993) }\end{array}$ & $\begin{array}{l}\text { Measure family } \\
\text { caregivers' } \\
\text { experience } \\
\text { with and } \\
\text { knowledge } \\
\text { about cancer } \\
\text { pain }\end{array}$ & None identified & $\begin{array}{l}\text { Family } \\
\text { caregivers of } \\
\text { cancer } \\
\text { patients }\end{array}$ & 13 & $\begin{array}{l}\text { Yes }(\alpha=0.81) / \\
\quad \text { yes }(\text { content })\end{array}$ \\
\hline $\begin{array}{l}\text { *Quality of Life in } \\
\text { Life-Threatening } \\
\text { Illness-Family } \\
\text { Carer Version } \\
\text { (QoLLTI-F) } \\
\text { (Cohen et al., } \\
\text { 2006) }\end{array}$ & QoL & $\begin{array}{l}\text { Carer's own state, } \\
\text { relationships, carer } \\
\text { outlook, quality of } \\
\text { care, patient } \\
\text { condition, finances, } \\
\text { environment }\end{array}$ & $\begin{array}{l}\text { Caregivers of } \\
\text { cancer } \\
\text { patients } \\
\text { receiving } \\
\text { palliative } \\
\text { care }\end{array}$ & 16 & $\begin{array}{c}\text { Yes }(\alpha=0.86) / \\
\text { yes }(\text { content, } \\
\text { construct) }\end{array}$ \\
\hline $\begin{array}{l}\text { CareGiver Oncology } \\
\text { Quality of Life } \\
\text { questionnaire } \\
\text { (CarGOQoL) } \\
\text { (Minaya et al., } \\
\text { 2012) }\end{array}$ & QoL & $\begin{array}{l}\text { Psychological well- } \\
\text { being, burden, } \\
\text { relationship with } \\
\text { healthcare, } \\
\text { administration and } \\
\text { finances, coping, } \\
\text { physical well-being, } \\
\text { self-esteem, leisure } \\
\text { time, social support } \\
\text { and private life }\end{array}$ & $\begin{array}{l}\text { Caregivers of } \\
\text { cancer } \\
\text { patients }\end{array}$ & 29 & $\begin{array}{l}\text { Yes }(0.72-0.89, \\
\text { except private } \\
\text { life } 0.55) / \text { yes } \\
\text { (construct, } \\
\text { convergent) }\end{array}$ \\
\hline $\begin{array}{l}\text { Caregiver Quality of } \\
\text { Life Index-Cancer } \\
\text { (Weitzner et al., } \\
1999 a \text { ) }\end{array}$ & QoL & None identified & $\begin{array}{l}\text { Primary } \\
\text { caregiver of } \\
\text { cancer } \\
\text { patients }\end{array}$ & 35 & $\begin{array}{l}\text { Yes }(\alpha=0.91) / \text { yes } \\
\text { (content, } \\
\text { construct, } \\
\text { convergent, } \\
\text { divergent) }\end{array}$ \\
\hline $\begin{array}{l}\text { City of Hope QoL } \\
\text { Scale-Family } \\
\text { Version (Ferrell } \\
\text { et al., 1991) }\end{array}$ & QoL & $\begin{array}{l}\text { Physical, psychological, } \\
\text { social, spiritual }\end{array}$ & $\begin{array}{l}\text { Family } \\
\text { caregivers of } \\
\text { cancer } \\
\text { patients }\end{array}$ & 37 & $\begin{array}{l}\text { Yes test-retest } \\
(0.89) \text { internal } \\
\text { consistency } \\
(\alpha=0.69) / \text { not } \\
\text { found }\end{array}$ \\
\hline $\begin{array}{l}\text { Quality of Life- } \\
\text { Family Caregiver } \\
\text { Tool (QoL) (Ferrell } \\
\text { et al., 1999) }\end{array}$ & QoL & $\begin{array}{l}\text { Physical, psychological, } \\
\text { social, spiritual }\end{array}$ & $\begin{array}{l}\text { Family } \\
\text { caregivers of } \\
\text { cancer } \\
\text { patients }\end{array}$ & 27 & $\begin{array}{l}\text { Yes }(\alpha=0.69) \\
\text { test-retest } \\
\quad(r=0.89) / \text { yes } \\
\text { (concurrent) }\end{array}$ \\
\hline $\begin{array}{l}\text { Pediatric Patients } \\
\text { Pediatric Quality of } \\
\text { Life Inventory } \\
\text { (PedsQL }{ }^{\mathrm{TM}} \text { ) Family } \\
\text { Impact Module } \\
\text { (Varni et al., 1999) }\end{array}$ & $\begin{array}{l}\text { QoL of parents } \\
\text { and family } \\
\text { functioning }\end{array}$ & $\begin{array}{l}6 \text { subscales measuring } \\
\text { parents' self-reported } \\
\text { functioning (physical } \\
\text { functioning, emotional } \\
\text { functioning, social } \\
\text { functioning, cognitive } \\
\text { functioning, } \\
\text { communication, } \\
\text { worry); } 2 \text { subscales } \\
\text { measuring parent- } \\
\text { reported family } \\
\text { functioning (daily } \\
\text { activities, family } \\
\text { relationships) }\end{array}$ & $\begin{array}{l}\text { Parents/ } \\
\text { guardians of } \\
\text { children with } \\
\text { cancer in } \\
\text { active therapy } \\
\text { aged } 2-18 \\
\text { years }\end{array}$ & 95 & $\begin{array}{l}\text { Yes (test-retest } \\
0.81- \\
0.96)(\alpha=0.73- \\
0.89) / \text { yes } \\
\text { (construct) }\end{array}$ \\
\hline
\end{tabular}


Table 4. Continued

\begin{tabular}{|c|c|c|c|c|c|}
\hline Instrument name & Purpose/focus & Domains/sections & $\begin{array}{c}\text { Original } \\
\text { population }\end{array}$ & $\begin{array}{l}\text { Number } \\
\text { of items }\end{array}$ & $\begin{array}{c}\text { Reliability/ } \\
\text { validity }\end{array}$ \\
\hline $\begin{array}{l}\text { Varricchio-Wright } \\
\text { Impact of Cancer } \\
\text { Scale-Parents } \\
\text { (VWICS-P) } \\
\text { (Wright, 1993) }\end{array}$ & QoL & None identified & $\begin{array}{l}\text { Parents of } \\
\text { children with } \\
\text { cancer }\end{array}$ & $\begin{array}{l}\text { Not } \\
\text { found }\end{array}$ & $\begin{array}{c}\text { Yes }(\alpha=0.67- \\
0.87) / \text { yes } \\
\text { (construct, } \\
\text { content) }\end{array}$ \\
\hline
\end{tabular}

*Self-report instruments with $\leq 20$ items, and positive reliability and validity.

\section{Definition of Need}

"Caregiver needs" have been defined as needs related to state of health and state of healthcare that are not being met (Hudson et al., 2010; Hileman \& Lackey, 1990).

\section{Definition of Quality of Life}

The Centers for Disease Control and Prevention (2015) defines "quality of life" (QoL) as a subjective evaluation of both the positive and negative aspects of life. QoL instruments typically measure psychological, social, and physical well-being but may also include one's spiritual state.

\section{Definition of Satisfaction with Care}

"Satisfaction" has been defined as the fulfillment of a need or want (Merriam-Webster, 2015). Satisfaction with care has been determined to be a significant factor, as the patient and caregiver will go through multiple aspects of care through the cancer trajectory (diagnosis, treatments, recurrence, survivorship, end of life), as well as the various healthcare specialties to which the patient will be referred.

Table 5. Instruments focusing on other aspects of caregiver distress

\begin{tabular}{|c|c|c|c|c|c|}
\hline Instrument name & Purpose/focus & Domains/sections & $\begin{array}{c}\text { Original } \\
\text { population }\end{array}$ & $\begin{array}{l}\text { Number } \\
\text { of items }\end{array}$ & $\begin{array}{l}\text { Reliability/ } \\
\text { validity }\end{array}$ \\
\hline \multicolumn{6}{|l|}{ Adult Patients } \\
\hline $\begin{array}{l}\text { Morbidities Index for } \\
\text { Caregivers of Chronic } \\
\text { Illnesses (MICCI) } \\
\text { (Kim et al., 2013) }\end{array}$ & $\begin{array}{l}\text { Caregiver } \\
\text { comorbidities }\end{array}$ & None identified & Cancer caregivers & 40 & No/no \\
\hline $\begin{array}{l}\text { Mutual Communal } \\
\text { Behaviors Scale } \\
\text { (Williamson \& Schulz, } \\
\text { 1995) }\end{array}$ & $\begin{array}{l}\text { Communal } \\
\text { feelings } \\
\text { between } \\
\text { caregiver and } \\
\text { patient }\end{array}$ & None identified & $\begin{array}{l}\text { Cancer caregivers } \\
\text { undergoing } \\
\text { outpatient } \\
\text { palliative } \\
\text { radiation } \\
\text { therapy }\end{array}$ & 10 & $\begin{array}{l}\text { Yes }(\alpha=0.86- \\
0.88 ; \text { test- } \\
\text { retest }=0.71 \\
0.67) / \text { not found }\end{array}$ \\
\hline \multicolumn{6}{|l|}{ Pediatric Patients } \\
\hline $\begin{array}{l}\text { Marwit and Meuser } \\
\text { Caregiver Inventory } \\
\text { Childhood Cancer } \\
\text { (MM-CGI Childhood } \\
\text { Cancer) (Al-Gamal } \\
\text { et al., 2009) }\end{array}$ & $\begin{array}{l}\text { Anticipatory } \\
\text { grief }\end{array}$ & $\begin{array}{l}\text { Personal sacrifice } \\
\text { burden, heartfelt } \\
\text { sadness and } \\
\text { longing, worry and } \\
\text { felt isolation }\end{array}$ & $\begin{array}{l}\text { Parents of } \\
\text { children with } \\
\text { cancer }\end{array}$ & 50 & $\begin{array}{l}\text { Yes }(\alpha=0.95) / \text { yes } \\
\quad(\text { construct })\end{array}$ \\
\hline $\begin{array}{l}\text { Parent Experience of } \\
\text { Child Illness (Bonner } \\
\text { et al., 2006) }\end{array}$ & $\begin{array}{l}\text { Parent } \\
\text { adjustment }\end{array}$ & $\begin{array}{l}\text { Guilt and worry, } \\
\text { unresolved sorrow } \\
\text { and anger, long- } \\
\text { term uncertainty, } \\
\text { emotional } \\
\text { resources }\end{array}$ & $\begin{array}{l}\text { Parents of } \\
\text { children with } \\
\text { brain tumors }\end{array}$ & 25 & $\begin{array}{c}\text { Yes }(\alpha=0.72- \\
0.89) / \text { yes } \\
\text { (construct) }\end{array}$ \\
\hline $\begin{array}{l}\text { SCREEM Family } \\
\text { Resources Survey } \\
\text { (SCREEM-RES) } \\
\text { (Panganiban-Corales } \\
\text { \& Medina, 2011) }\end{array}$ & Family resources & $\begin{array}{l}\text { Social, cultural, } \\
\text { religious, } \\
\text { economic, } \\
\text { educational, } \\
\text { medical resources }\end{array}$ & $\begin{array}{l}\text { Family caregivers } \\
\text { of children with } \\
\text { cancer }\end{array}$ & 12 & $\begin{array}{l}\text { Yes }(\alpha=0.80) / \text { not } \\
\quad \text { found }\end{array}$ \\
\hline
\end{tabular}


Table 6. Breakdown of number of items per instrument

\begin{tabular}{lr}
\hline \hline Number of items & $n$ \\
\hline $\mathbf{1 - 1 0}$ & 3 \\
$\mathbf{1 1 - 2 0}$ & 17 \\
$\mathbf{2 1 - 3 0}$ & 13 \\
$\mathbf{3 1 - 4 0}$ & 9 \\
$\mathbf{4 1 - 5 0}$ & 8 \\
$\mathbf{5 1 - 1 0 0}$ & 8 \\
$\mathbf{7 1 0 0}$ & 1 \\
Total & 59 \\
\hline \hline
\end{tabular}

\section{Definition of Other Aspects}

"Other aspects" were defined as any other aspect in caregiving that did not fit exclusively into any of the previous categories, including emotions between caregivers and patients, caregiver comorbidity, and anticipatory grief.

\section{RESULTS}

The database search identified 5,541 articles. These were narrowed down to 135 articles $(2.4 \%)$ based on our inclusion criteria. All 135 were extensively discussed by the authors, and a consensus was reached. From these we extracted 59 instruments that measured different dimensions of cancer caregiving distress. Instruments that had similar names to other tools were labeled according to their country/region of origin. The abbreviated names of the instruments were also listed. The PRISMA diagram is shown in Supplementary Appendix B.

The instruments were divided into the following categories: burden $(n=26,44 \%)$; needs $(n=14$, $23.7 \%$ ); quality of life ([QoL] $n=9,15.3 \%$ ); satisfaction with healthcare delivery $(n=5,8.5 \%)$; and various other issues $(n=5 ; 8.5 \%)$. Among the 59 instruments, 45 (74.6\%) measured caregiver distress in adult cancer patients and $15(25.4 \%)$ in caregivers of pediatric patients. Forty-one of the $(69.5 \%)$ instruments were found to be reliable and valid. The list of instruments and associated information are presented in Tables $1-5$.

The median number of items was 29 (4-125). Twenty instruments $(33.9 \%)$ had $\leq 20$ items. There were 9 instruments that had $\geq 50$ items (15.3\%). The breakdown of number of items is shown in Table 6 . Thirteen instruments $(22 \%)$ had $\leq 20$ items and were valid and reliable, $12(92.3 \%)$ of which were self-report instruments. These instruments are denoted in bold and shown first in Tables 1-5.

Of the caregiver burden instruments, 9 of the 26 $(34.6 \%)$ had $\leq 20$ items, with $5(55.6 \%)$ being reliable, valid, and self-report. Eighteen $(69.2 \%)$ of the tools measured caregiver burden in adult patients. Of the caregiver needs instruments, 3 of $14(21.4 \%)$ were reliable and valid, and had $\leq 20$ items, with 1 of these $3(33.3 \%)$ requiring administration by healthcare providers. Twelve of the 14 (85.7\%) needs instruments measured the caregiver needs of adult patients. Of the instruments measuring satisfaction with care, 2 of 5 (40\%) were self-report, reliable, and valid and had $\leq 20$ items. All instruments for caregiver satisfaction were for caregivers of adult patients. Of the caregiver QoL instruments, 3 of 9 $(33.3 \%)$ were self-report, reliable, and valid and had $\leq 20$ items. Seven of the $9(77.8 \%)$ measured caregiver $\mathrm{QoL}$ in adult patients.

\section{DISCUSSION}

Our systematic review of the literature identified 135 of 5,541 articles that met our inclusion criteria, with 59 instruments found to be developed initially for caregivers of cancer patients. Some of these instruments were mentioned in multiple articles, thereby explaining the larger number of articles than instruments.

As a general comment, we found it difficult to document whether a particular tool was developed for research or clinical purposes. Instead, we included the number of items in each instrument to indirectly illustrate if the tool was too complex or time-consuming to complete during a regular healthcare visit. Among all instruments, about $20 \%$ were reliable, valid, had $\leq 20$ items, and were self-report questionnaires. We believe this is an encouraging number of instruments that are psychometrically sound and, more importantly, that can be applied effectively in a clinical setting. Among these instruments, McMillan's Caregiver Quality of Life Index appeared to be the simplest questionnaire, with four items measuring a caregiver's physical, emotional, social, and financial well-being (McMillan \& Mahon, 1994). Although interesting for research purposes in understanding the complexities of caregiver distress, lengthy instruments can place a heavy burden on already-stressed caregivers and, likewise, may increase the burden on clinicians in trying to apply these instruments in a busy clinical setting. Self-report instruments may also help decrease the burden on busy healthcare providers, as caregivers are able to complete them without taking time away from providers.

Our review demonstrated a considerable number of instruments measuring caregiver burden $(n=26)$ and caregiver needs $(n=14)$. While Deeken et al. (2003), in their review of self-report instruments for informal caregivers, appropriately identified the 
disproportion of quality-of-life tools between cancer patients and caregivers, our search was actually able to identify a higher number of caregiver QoL tools compared to previous literature reviews (Hudson et al., 2010; Deeken et al., 2003). On the contrary, there is a dearth of instruments measuring family satisfaction with care, grief, and bereavement.

We found that there were 44 of 59 (74.6\%) adult patient-based cancer caregiver instruments compared to $15(25.4 \%)$ pediatric patient-based cancer caregiver instruments, which is not surprising when compared to overall cancer statistics (Murphy et al., 2013; Howlader et al., 2013; NIH, 2015). Although a statistical analysis suggests that cancer is overwhelmingly a disease of adults, the diagnosis of cancer in a child puts enormous burden on caregivers, so that assessment tools are of great importance in that population (Sargent et al., 1995; Woodgate, 2006; Patterson et al., 2004). Furthermore, there are very specific issues in pediatric caregiving, including the effects of advanced disease on children and adolescents, and those need to be addressed in future research.

One of the limitations of the study is that we did not address tools that were not designed initially for cancer caregivers but can and are being used currently for that population. We believe that the level of complexity of caring for cancer patients may need specifically designed tools to capture their level of distress. There were several valuable tools that were excluded from the final tally. These tools were initially developed for other diseases or for patients, and were then eventually found to be applicable for caregivers as well. Some of these tools included the Zarit Burden Interview, developed for caregivers of dementia patients (Zarit et al., 1980); the Distress Thermometer, initially utilized with prostate cancer patients (Roth et al., 1998); and the Family Relationships Index, initially used in cancer patients and was part of the Family Environment Scale (Edwards \& Clarke, 2005). Although not meeting the inclusion criteria for our review, these tools should not be neglected and are particularly valuable in detecting distress for both caregivers and patients with different disease processes.

Another limitation is the choice of 20 items as a cutoff for the instruments, which we based on our clinical experience in using other regular clinical assessment tools, including the Edmonton Symptom Assessment System (ESAS) and the Memorial Delirium Assessment Scale (MDAS). It might be possible that certain lengthier instruments would also be feasible and time-efficient in the clinical setting.

As compared to the reviews conducted by Deeken et al. (2003), Hudson et al. (2010), and Applebaum and Breitbart (2012), our research supports their findings and also adds more instruments to the list that can be utilized for caregivers of adults and pediatric cancer patients. We also confirmed that there is a significant number of tools that have $\leq 20$ items and that can be applied clinically. The challenge for the future is how to implement them regularly, improving caregiver care and measuring outcomes in the adaptation of these tools. Since there is no gold standard or real comparison between the different tools, clinical teams will have to determine which of these tools are more suitable to their practice. Ideally, in the future, receiving caregiver feedback will help identify the most effective.

Our review of tools has demonstrated that very few instruments have been incorporated into regular clinical practice. In addition, there are no studies documenting how the use of these tools helps modify clinical and/or health service utilization outcomes. There is also a lack of data on the comparative clinical performance of these instruments. These are all areas where research is needed.

We have found that there are several self-report cancer caregiver distress instruments that are concise, reliable, and valid. Clinical utilization and outcomes measures are certainly important areas for future research.

\section{SUPPLEMENTARY MATERIALS AND METHODS}

To view supplementary material for this article, please visit http://dx.doi.org/10.1017/S1478951516000079.

\section{REFERENCES}

Al-Gamal, E., Long, T. \& Livesley, J. (2009). Development of a modified instrument to measure anticipatory grieving in Jordanian parents of children diagnosed with cancer: The Marwit and Meuser Caregiver Inventory Childhood Cancer. Cancer Nursing, 32, 211-219.

Applebaum, A.J. \& Breitbart, W. (2012). Care for the cancer caregiver: A systematic review. Palliative \& Supportive Care, 11, 231-252.

Aslett, H., Morrison, V., Zinovieff, F., et al. (2009). It's not what you ask, but how you ask it: Needs disclosure by familial carers of cancer patients. Psycho-Oncology, $18, \mathrm{~S} 2$

Bachner, Y.G., Gesis, Z., Davidov, E., et al. (2008). Caregivers' communication with patients about illness and death: Initial validation of a scale. Omega (Westport), 57(4), 381-397.

Bonner, M.J., Hardy, K.K., Guill, A.B., et al. (2006). Development and validation of the parent experience of child illness. Journal of Pediatric Psychology, 31(3), 310-321.

Cameron, J.I., Franche, R.L., Cheung, A.M., et al. (2002). Lifestyle interference and emotional distress in family caregivers of advanced cancer patients. Cancer, 94(2), $521-527$. 
Campbell, H.S., Sanson-Fisher, R., Taylor-Brown, J., et al. (2009). The cancer support person's unmet needs survey: Psychometric properties. Cancer, 115, 3351-3359.

Casarett, D., Pickard, A., Bailey, F.A., et al. (2008). Do palliative consultations improve patient outcomes? Journal of the American Geriatric Society, 56(4), 593-599.

Centers for Disease Control and Prevention (2015). Healthrelated quality of life (HRQoL). Available from http:// www.cdc.gov/hrqol/concept.htm. Accessed October 7, 2015.

Chen, H.C., Chen, M.L., Lotus Shyu, Y.I., et al. (2007). Development and testing of a scale to measure caregiving load in caregivers of cancer patients in Taiwan: The Care Task Scale-Cancer. Cancer Nursing, 30(3), $223-231$.

Christakis, N.A. \& Allison, P.D. (2006). Mortality after the hospitalization of a spouse. The New England Journal of Medicine, 354, 719-730.

Cohen, R., Leis, A.M., Kuhl, D., et al. (2006). QoLLTI-F: Measuring family carer quality of life. Palliative Medicine, 20, 755-767.

Cooper, B., Kinsella, G.J. \& Picton, C. (2006). Development and initial validation of a family appraisal of caregiving questionnaire for palliative care. Psycho-Oncology, 15(7), 613-622.

Deeken, J.F., Taylor, K.L., Mangan, P., et al. (2003). Care for the caregivers: A review of self-report instruments developed to measure the burden, needs, and quality of life of informal caregivers. Journal of Pain and Symptom Management, 26(4), 922-953.

Dumont, S., Turgeon, J., Allard, P., et al. (2006). Caring for a loved one with advanced cancer: Determinants of psychological distress in family caregivers. Journal of Palliative Medicine, 9, 912-921.

Duran, B. (2011). Developing a scale to measure parental worry and their attitudes toward childhood cancer after successful completion of treatment: A pilot study. Journal of Pediatric Oncology Nursing, 28(3), 154-168.

Edwards, B. \& Clarke, V. (2005). The validity of the family relationships index as a screening tool for psychological risk in families of cancer patients. Psycho-Oncology, 14(7), 546-554.

Enskar, K., Carlsson, M., von Essen, L., et al. (1997). Development of a tool to measure the life situation of parents of children with cancer. Quality of Life Research, 6, $248-256$.

Ewing, G. \& Grande, G. (2013). Development of a Carer Support Needs Assessment Tool (CSNAT) for end-oflife care practice at home: A qualitative study. Palliative Medicine, 27, 244-256.

Ferrell, B.R., Ferrell, B.A., Rhiner, M., et al. (1991). Family factors influencing cancer pain management. Postgraduate Medical Journal, 67(Suppl. 2), S64-S69.

Ferrell, B., Rhiner, M. \& Rivera, L.M. (1993). Development and evaluation of the Family Pain Questionnaire. Journal of Psychosocial Oncology, 10(4), 21-36.

Ferrell, B.R., Grant, M., Bornerman, T., et al. (1999). Family caregiving in cancer pain management. Journal of Palliative Medicine, 2, 185-195.

Fried, T.R., Bradley, E.H. \& Towle, V.R. (2003). Valuing the outcomes of treatment: Do patients and their caregivers agree? Archives of Internal Medicine, 163(17), 2073-2078.

Gelfman, L.P., Meier, D.E. \& Morrison, R.S. (2008). Does palliative care improve quality? A survey of bereaved family members. Journal of Pain and Symptom Management, 36(1), 22-28.
Girgis, A., Lambert, S. \& Lecathelinais, C. (2011). The supportive care needs survey for partners and caregivers of cancer survivors: Development and psychometric evaluation. Psycho-Oncology, 20, 387-393.

Given, B., Wyatt, G., Given, C., et al. (2004). Burden and depression among caregivers of patients with cancer at the end of life. Oncology Nursing Forum, 31, 1105-1117.

Given, C.W., Given, B., Stommel, M., et al. (1992). The caregiver reaction assessment (CRA) for caregivers to persons with chronic physical and mental impairments. Research in Nursing \& Health, 15, 271-283.

Glajchen, M. (2009). Role of family caregivers in cancer pain management. In Cancer pain: Assessment and management, 2nd ed. E.D. Bruera \& R.K. Portenoy (eds.), pp. 597-607. New York: Cambridge University Press.

Glajchen, M., Kornblith, A., Homel, P., et al. (2005). Development of a brief assessment scale for caregivers of the medically ill. Journal of Pain and Symptom Management, 29, 245-254.

Gwyther, L.P. \& George, L.K. (1986). Symposium. Caregivers for dementia patients: Complex determinants of well-being and burden. The Gerontologist, 26, 245-247.

Haberman, M.R., Woods, N.F. \& Packard, N.J. (1990). Demands of chronic illness: Reliability and validity assessment of a demands-of-illness inventory. Holistic Nursing Practice, 6, 25-35.

Hearson, B. \& Clement, S. (2007). Sleep disturbance in family caregivers of patients with advanced cancer. International Journal of Palliative Nursing, 13, 495-501.

Heyland, D.K., Cook, D.J., Rocker, G.M., et al. (2010). The development and validation of a novel questionnaire to measure patient and family satisfaction with end-oflife care: The Canadian Health Care Evaluation Project (CANHELP) Questionnaire. Palliative Medicine, 24, 682-695.

Higgins, P.C. \& Prigerson, H.G. (2013). Caregiver evaluation of the quality of end-of-life care (CEQUEL) scale: The caregiver's perception of patient care near death. PLoS One, 8(6), e66066.

Hileman, J.W. \& Lackey, N.R. (1990). Self-identified needs of patients with cancer at home and their home caregivers: A descriptive study. Oncology Nursing Forum, 17(6), 907-913.

Hileman, J.W., Lackey, N.R. \& Hassanein, R.S. (1992). Identifying the needs of home caregivers of patients with cancer. Oncology Nursing Forum, 19, 771-777.

Howlader, N., Noone, A.M., Krapcho, M., et al., (eds.) (2013). Cancer statistics review, 1975-2010. Bethesda, MD: National Cancer Institute.

Hudson, P.L., Trauer, T., Graham, S., et al. (2010). A systematic review of instruments related to family caregivers of palliative care patients. Palliative Medicine, 24(7), 656-668.

Ishii, Y., Miyashita, M., Sato, K., et al. (2012). Family's difficulty scale in end-of-life home care: A new measure of the family's difficulties in caring for patients with cancer at the end of life at home from bereaved family's perspective. Journal of Palliative Medicine, 15(2), $210-215$.

Kang, K.A. (2011). Reliability and validity of the suffering scale of family of patients with terminal cancer [in Korean]. Journal of Korean Oncology Nursing, 11(1), 49-57.

Kazak, A.E., Prusak, A., McSherry, M., et al. (2001). The Psychosocial Assessment Tool (PAT): Pilot data on a brief screening instrument for identifying high risk 
families in pediatric oncology. Families, Systems, \& Health , 19, 303-317.

Kazak, A.E., McClure, K.S., Alderfer, M.A., et al. (2004). Cancer-related parental beliefs: The Family Illness Beliefs Inventory (FIBI). Journal of Pediatric Psychology, 29, 531-542.

Kilpatrick, M.G., Kristjanson, L.J. \& Tataryn, D.J. (1998). Measuring the information needs of husbands of women with breast cancer: Validity and reliability of the Family Inventory of Needs-Husbands. Oncology Nursing Forum, 25, 1347-1351.

Kim, Y. \& Given, B.A. (2008). Quality of life of family caregivers of cancer survivors. Cancer, 112, 2556-2568.

Kim, Y., Wellisch, D.K., Spillers, R.L., et al. (2007). Psychological distress of female cancer caregivers: Effects of type of cancer and caregivers' spirituality. Supportive Care in Cancer, 15(12), 1367-1374.

Kim, Y., Kashy, D., Spillers, R., et al. (2010). Needs assessment of family caregivers of cancer survivors: Three cohorts comparison. Psycho-Oncology, 19, 573-582.

Kim, Y., Carver, C.S., Cannady, R.S., et al. (2013). Selfreported medical morbidity among informal caregivers of chronic illness: The case of cancer. Quality of Life Research, 22, 1265-1272.

Kristjanson, L.J. (1993). Validity and reliability testing of the FAMCARE scale: Measuring family satisfaction with advanced cancer care. Social Science \& Medicine, $36,693-701$.

Kristjanson, L.J., Atwood, J. \& Degner, L.F. (1995). Validity and reliability of the family inventory of needs (FIN): Measuring the care needs of families of advanced cancer patients. Journal of Nursing Measurement, 3, 109-126.

Levesque, J.V. \& Maybery, D.J. (2014). The Parental Cancer Questionnaire: Scale structure, reliability, and validity. Supportive Care in Cancer, 22(1), 23-32.

Longman, A.J., Atwood, J.R., Sherman, J.B., et al. (1992). Care needs of home-based cancer patients and their caregivers: Quantitative findings. Cancer Nursing, 15(3), 182-190.

Lund, L., Ross, L. \& Groenvold, M. (2012). The initial development of the Cancer Caregiving Tasks, Consequences and Needs Questionnaire (CaTCoN). Acta Oncologica, 51(8), 1009-1019.

McMillan, S.C. \& Mahon, M. (1994). The impact of hospice services on the quality of life of primary caregivers. Oncology Nursing Forum, 21, 1189-1195.

Merriam-Webster (2015). Definition of "satisfaction." Available from http://www.merriam-webster.com/ dictionary/satisfaction.

Minaya, P., Baumstarck, K., Berbis, J., et al. (2012). The Caregiver Oncology Quality of Life Questionnaire (CarGOQoL): Development and validation of an instrument to measure the quality of life of the caregivers of patients with cancer. European Journal of Cancer, 48(6), 904-911.

Mitchell, G., Girgis, A., Moyez, J., et al. (2010). A GP caregiver needs toolkit versus usual care in the management of the needs of caregivers of patients with advanced cancer: A randomized controlled trial. Trials, 11, 115.

Miyashita, M., Morita, T., Sato, K., et al. (2008). Good death inventory: A measure for evaluating a good death from the bereaved family member's perspective. Journal of Pain and Symptom Management, 35(5), 486-498.

Monterosso, L., Kristjanson, L.J. \& Dadd, G. (2006). Content validity and reliability testing of the FIN-PED II: A tool to measure care needs of parents of children with cancer. Journal of Nursing Measurement, 14(1), $31-44$.

Montgomery, R.J.V., Gonyea, J.G. \& Hooyman, N.R. (1985). Caregiving and the experience of subjective and objective burden. Family Relations, 34, 19-26.

Murphy, S.L., Xu, J. \& Kochanek, K.D. (2013). Deaths: Final data for 2010. National Vital Statistics Reports, Vol. 61, No. 4. Hyattsville, MD: National Center for Health Statistics.

National Cancer Institute (2015). Family caregivers in cancer: Roles and challenges. Available from http://www. cancer.gov/cancertopics/pdq/supportivecare/caregivers/ healthprofessional/page1/AllPages\#Reference2.9.

National Institutes of Health (NIH) (2015). SEER cancer stat fact sheets (all sites). Available from http://seer. cancer.gov/statfacts/html/all.html.

Nolan, M.T., Hughes, M.T., Kub, J., et al. (2009). Development and validation of the Family Decision-Making Self-Efficacy Scale. Palliative \& Supportive Care, 7, $315-321$.

Oberst, M.T., Thomas, S.E., Gass, K.A., et al. (1989). Caregiving demands and appraisal of stress among family caregivers. Cancer Nursing, 12, 209-215.

Pai, A., Patino-Fernandez, A.M., McSherry, M., et al. (2008). The Psychosocial Assessment Tool (PAT 2.0): Psychometric properties of a screener for psychosocial distress in families of children newly diagnosed with cancer. Journal of Pediatric Psychology, 33, 50-62.

Panganiban-Corales, A.T. \& Medina, M.F. (2011). Family resources study, part 1: Family resources, family function and caregiver strain in childhood cancer. Asia Pacific Family Medicine, 10(1), 10-14.

Partinico, M., Cora, A., Ghisi, M., et al. (2014). A new Italian questionnaire to assess caregivers of cancer patients' satisfaction with palliative care: Multicenter validation of the post mortem questionnaire-short form. Journal of Pain and Symptom Management, 47(2), 298-306.

Patterson, J.M., Holm, K.R. \& Gurney, J.G. (2004). The impact of childhood cancer on the family: A qualitative analysis of strains, resources, and coping behaviors. Psycho-Oncology, 13, 390-407.

Patterson, P., Pearce, A. \& Slawitschka, E. (2011). The initial development of an instrument to assess the psychosocial needs and unmet needs of young people who have a parent with cancer: Piloting the offspring cancer needs instrument (OCNI). Supportive Care in Cancer, 19(8), 1165-1174.

Roth, A.J., Kornblith, A.B., Batel-Copel, L., et al. (1998). Rapid screening for psychologic distress in men with prostate carcinoma: A pilot study. Cancer, 82(10), 1904-1908.

Sales, E. (1992). Psychosocial impact of the phase of cancer on the family: An updated review. Journal of Psychosocial Oncology, 9(4), 1-18.

Sanjo, M., Morita, T., Miyashita, M., et al. (2009). Caregiving Consequences Inventory: A measure for evaluating caregiving consequences from the bereaved family member's perspective. Psycho-Oncology, 18(6), 657-666.

Sargent, J.R., Sahler, O.J.Z., Roghmann, K.J., et al. (1995). Sibling adaptation to childhood cancer collaborative study: Siblings' perceptions of the cancer experience. Journal of Pediatric Psychology, 20(2), 151-164.

Siminoff, L.A., Rose, J.H., Zhang, A., et al. (2006). Measuring discord in treatment decision-making: Progress toward development of a cancer communication and 
decision-making assessment tool. Psycho-Oncology, 15(6), 528-540.

Sinfield, P., Baker, R., Tarrant, C., et al. (2009). The Prostate Care Questionnaire for Carers (PCQ-C): Reliability, validity and acceptability. BMC Health Services Research, 9, 229.

Streisand, R., Braniecki, S., Tercyak, K.P., et al. (2001). Childhood illness-related parenting stress: The pediatric inventory for parents. Journal of Pediatric Psychology, 26, 155-162.

Teel, C.S. \& Press, A.N. (1999). Fatigue among elders in caregiving and non-caregiving roles. Western Journal of Nursing Research, 21, 498-520.

Tringali, C. (1986). The needs of family members of cancer patients. Oncology Nursing Forum, 13(4), 65-70.

Ugalde, A., Krishnasamy, M. \& Schofield, P. (2013). Development of an instrument to self-efficacy in caregivers of people with advanced cancer. Psycho-Oncology, 22(6), 1428-1434.

Varni, J.W., Seid, M. \& Rode, C.A. (1999). The PedsQL: Measurement model for the pediatric quality of life inventory. Medical Care, 37(2), 126-139.

von Kanel, R., Mausbach, B.T., Patterson, T.L., et al. (2008). Increased Framingham Coronary Heart Disease Risk Score in dementia caregivers relative to non-caregiving controls. Gerontology, 54, 131-137.

Waller, A., Girgis, A., Currow, D., et al. (2008). Development of the Palliative Care Needs Assessment Tool (PC-NAT) for use by multi-disciplinary health professionals. Palliative Medicine, 22, 956-964.

Ward, S.E., Berry, P.E. \& Misiewicz, H. (1996). Concerns about analgesics among patients and family caregivers in a hospice setting. Research in Nursing \& Health, 19(3), 205-211.

Weitzner, M.A., Jacobsen, P.B., Wagner, H. Jr., et al. (1999a). The Caregiver Quality of Life Index-Cancer (CQoLC) scale: Development and validation of an instrument to measure quality of life of the family caregiver of patients with cancer. Quality of Life Research, 8, 55-63.

Weitzner, M.A., McMillan, S.C. \& Jacobsen, P.B. (1999b). Family caregiver quality of life: Differences between curative and palliative cancer treatment settings. Journal of Pain and Symptom Management, 17(6), 418-428.

Wells, D.K., James, K., Stewart, J., et al. (2002). The Care of My Child with Cancer: A new instrument to measure caregiving demand in parents of children with cancer. Journal of Pediatric Nursing, 17, 201-210.

Whiteley, E.M.J., Kristjanson, L.J., Degner, L.F., et al. (1999). Measuring the care needs of mothers of children with cancer: Development of the FIN-PED. The Canadian Journal of Nursing Research, 31, 103-123.

Williamson, G.M. \& Schulz, R. (1995). Caring for a family member with cancer: Past communal behavior and affective reactions. Journal of Applied Social Psychology, $25,93-116$.

Woodgate, R.L. (2006). Siblings' experiences with childhood cancer: A different way of being in the family. Cancer Nursing, 29(5), 406-414.

Wright, P.S. (1993). Parents' perceptions of their quality of life. Journal of Pediatric Oncology Nursing, 10(4), $139-145$.

Wu, K.K., Cho, V.W., Li, A., et al. (2010). Development of a psychological well-being scale for family caregivers in palliative care. East Asian Archives of Psychiatry, 20(3), 109-115.

Yabroff, K.R., Davis, W.W., Lamont, E.B., et al. (2007). Patient time costs associated with cancer care. Journal of the National Cancer Institute, 22(1), 14-23.

Yeh, C.H. (2001). Development and testing of the Parental Coping Strategy Inventory (PCSI) with children with cancer in Taiwan. Journal of Advanced Nursing, 36, $78-88$.

Zarit, S.H., Reever, K.E. \& Bach-Peterson, J. (1980). Relatives of the impaired elderly: Correlates of feelings of burden. The Gerontologist, 20, 649-655. 